\title{
ON PARABOLIC CLOSURES IN COXETER GROUPS
}

\author{
MATTHEW DYER
}

\begin{abstract}
For a finitely generated subgroup $W^{\prime}$ of a Coxeter system $(W, S)$, there are finitely generated reflection subgroups $W_{1}, \ldots, W_{n}$ of $W$, each containing $W^{\prime}$, such that any reflection subgroup of $W$ containing $W^{\prime}$ contains one of the $W_{i}$ as a standard parabolic subgroup. The canonical Coxeter generators of the $W_{i}$, and an expression for the parabolic closure of $W^{\prime}$ as a $W$-conjugate of a standard parabolic subgroup of $W$, may be effectively determined.
\end{abstract}

\section{INTRODUCTION AND STATEMENT OF RESULTS}

This note describes results on the structure of reflection subgroups of a Coxeter system $(W, S)$ which together afford an algorithm for computing the parabolic closure of (i.e. the parabolic subgroup of minimal rank containing) a given finitely generated subgroup of $W$.

Recall that the standard parabolic subgroups of $W$ are the subgroups $W_{J}=\langle J\rangle$ generated by subsets $J$ of $S$, and the parabolic subgroups of $W$ are the $W$-conjugates of the standard parabolic subgroups; these notions depend on $S$. Any reflection subgroup $W^{\prime}$ of $W$ has a canonical set of Coxeter generators (depending on $S$ ) which we denote as $\chi\left(W^{\prime}\right)$. Notions of parabolic and standard parabolic subgroups, rank etc of $W^{\prime}$ are defined in terms of the Coxeter generators $\chi\left(W^{\prime}\right)$ of $W^{\prime}$.

In Section 2, we provide more background on the above notions, and prove the following results.

Proposition 1. Let $W^{\prime}$ be a finitely generated subgroup of $W$. Then there exist $n \in \mathbb{N}_{>1}$ and finitely generated reflection subgroups $W_{1}, \ldots, W_{n}$ of $W$ such that each $W_{i}$ contains $W^{\prime}$ and if $W^{\prime \prime}$ is a reflection subgroup of $W$ with $W^{\prime \prime} \supseteq W^{\prime}$, then $W^{\prime \prime}$ contains $W_{i}$ as a standard parabolic subgroup for some $i$ with $1 \leq i \leq n$.

Proposition 2. Let $W^{\prime \prime}$ be a finitely generated reflection subgroup of $W$ with $\chi\left(W^{\prime \prime}\right)=\left\{c_{1}, \ldots, c_{n}\right\}$ where $n=\left|\chi\left(W^{\prime \prime}\right)\right|$. Choose a finite subset $J$ of $S$ such that $W^{\prime \prime} \subseteq W_{J}$. Then $W^{\prime \prime}$ is a parabolic subgroup of Wiff there exist pairwise distinct $s_{1}, \ldots, s_{n} \in J$ and some $w \in W_{J}$ with $w\left(c_{1} \cdots c_{n}\right) w^{-1}=s_{1} \cdots s_{n}$, in which case $W^{\prime \prime}=w^{-1} W_{\left\{s_{1}, \ldots, s_{n}\right\}} w$.

Corollary 3. The parabolic closure of the finitely generated subgroup $W^{\prime}$ of $W$ is the (unique) subgroup $W_{i}$ in Proposition 1 which is of minimal rank among the subgroups $W_{j}$, for $j=1, \ldots, n$, which are parabolic in $W$.

The proof of Proposition 1 shows that the sets of canonical Coxeter generators $\chi\left(W_{i}\right)$ can be effectively determined from a finite set of generators of $W^{\prime}$. Proposition 2 provides an effective test for determining whether a finitely-generated reflection subgroup of $W$ is parabolic, since it involves only finitely many tests for 
conjugacy of elements of the finitely-generated Coxeter group $W_{J}$, and the conjugacy problem for (finitely generated) Coxeter groups is solvable in general (see [5], [1]; in fact, it is known [10] that for any finitely generated Coxeter system $(W, S)$, there is a computable constant $N \in \mathbb{N}$ such that if $x, y \in W$ are $W$-conjugate, there is an element $w \in W$ of length $l(w) \leq N(l(x)+l(y))$ with $\left.y=w x w^{-1}\right)$. An explicit expression of the parabolic closure of $W^{\prime}$ as a $W$-conjugate of a standard parabolic subgroup of $W$ may therefore be effectively determined from a finite set of generators of $W^{\prime}$ using Corollary 3 and Proposition 2.

We remark that a quite different algorithm for computing the parabolic closure of a cyclic subgroup of $W$ was given in [10], where it was used as a preliminary step in various polynomial time algorithms for solving the conjugacy problem. Despite Proposition 2, an effective test for conjugacy of finitely-generated reflection subgroups of $W$ in general is not known to the author.

\section{BACKGROUND AND PROOF OF RESULTS}

As general references for facts on Coxeter groups and their reflection representations, root systems, Bruhat order etc used here, see [2] and [9]

2.1. Let $(W, S)$ be a Coxeter system, $l: W \rightarrow \mathbb{N}$ denote its standard length function, and $T=\left\{w s w^{-1} \mid w \in W, s \in S\right\}$ denote its set of reflections. For $w \in W$, let $N(w):=\{t \in T \mid l(t w)<l(w)\}$. Let $\leq$ denote Bruhat order on $W$, and $e=1_{W}$.

A subgroup $W^{\prime}$ of $W$ is called a reflection subgroup if it is generated by $W^{\prime} \cap T$. Let $W^{\prime}$ be a reflection subgroup of $W$. Then by [7] or [6], $W^{\prime}$ has a canonical set of Coxeter generators $S^{\prime}=\chi(W):=\left\{t \in T \mid N(t) \cap W^{\prime}=\{t\}\right\}$. We say a subgroup of $W^{\prime}$ is a standard parabolic subgroup of $W^{\prime}$ if it is generated by a subset of $\chi\left(W^{\prime}\right)$. A subgroup of $W^{\prime}$ is called a parabolic subgroup of $W^{\prime}$ if it is conjugate in $W^{\prime}$ to a standard parabolic subgroup of $W^{\prime}$. An algorithm for computing $\chi\left(W^{\prime}\right)$ from a finite set of reflections generating $W^{\prime}$ is described in [7] and in more detail in [6]. The cardinality of $\chi\left(W^{\prime}\right)$ will be called the rank of $W^{\prime}$.

2.2. Here, we recall some general facts from [8]. Let $\Omega_{(W, S)}$ be the directed graph with vertex set $W$ and with set of directed edges $\{(t w, w) \mid w \in W, t \in N(w)\}$, where we view $(t w, w)$ with $t \in N(w)$ as an edge directed from $t w$ to $w$. For $A \subseteq W$, let $\Omega_{(W, S)}(A)$ denote the full subgraph of $\Omega_{(W, S)}$ with vertex set $A$. Another characterization of $S^{\prime}=\chi\left(W^{\prime}\right)$ amongst sets of Coxeter generators of $W^{\prime}$ is that for any $x \in W$, there is an element $y \in x W^{\prime}$ such that the map $\Omega_{(W, S)}\left(x W^{\prime}\right) \rightarrow \Omega_{\left(W^{\prime}, S^{\prime}\right)}$ given by $w \mapsto y^{-1} w$ an isomorphism of directed graphs. The element $y$ is the unique element of $x W^{\prime}$ with $N\left(y^{-1}\right) \cap W^{\prime}=\emptyset$, the unique element of minimal length $l(y)$ in the coset $x W^{\prime}$, the unique source of the graph $\Omega_{(W, S)}\left(x W^{\prime}\right)$ and the unique element $y \in x W^{\prime}$ such that there is no edge $(y r, y)$ in $\Omega_{(W, S)}$ with $r \in \chi\left(W^{\prime}\right)$. It is easy to see from the last description of $y$ that if $W^{\prime}$ is finitely generated (i.e. $\chi\left(W^{\prime}\right)$ is finite) then $y$ is effectively computable from $x$ and $\chi\left(W^{\prime}\right)$.

For use in the proof of Proposition 2, we record the following:

Lemma 1. Let $W^{\prime \prime}$ be a reflection subgroup of $W$ and $w \in W$. Fix $u \in w W^{\prime \prime}$ with $N\left(u^{-1}\right) \cap W^{\prime \prime}=\emptyset$. Then $\chi\left(w W^{\prime \prime} w^{-1}\right)=u \chi\left(W^{\prime \prime}\right) u^{-1}$.

Proof. Let $S^{\prime \prime}:=\chi\left(W^{\prime \prime}\right)$. Now $w W^{\prime \prime} w^{-1}=u W^{\prime \prime} u^{-1}$ clearly has $u S^{\prime \prime} u^{-1}$ as a set of generators. Since the set $\chi\left(u W^{\prime \prime} u^{-1}\right)$ of Coxeter generators of $u W^{\prime \prime} u^{-1}$ is a minimal set of generators of $u W^{\prime \prime} u^{-1}$, it will suffice to show that $u S^{\prime \prime} u^{-1} \subseteq \chi\left(u W^{\prime \prime} u^{-1}\right)$. 
Let $s \in S^{\prime \prime}$ i.e. $s \in T$ with $N(s) \cap W^{\prime \prime}=\{s\}$. To prove $u s u^{-1} \in \chi\left(u W^{\prime \prime} u^{-1}\right)$, we must show $N\left(u s u^{-1}\right) \cap u W^{\prime \prime} u^{-1}=\left\{u s u^{-1}\right\}$ or $u^{-1} N\left(u s u^{-1}\right) u \cap W^{\prime \prime}=\{s\}$. To show this, regard $N$ as a cocycle of $W$ with values in the power set of $T$, regarded as additive abelian group under symmetric difference and with left $W$-action by conjugation, as in [7].

The cocycle condition gives $N\left(u s u^{-1}\right)=N(u)+u N(s) u^{-1}+u s N\left(u^{-1}\right) s u^{-1}$. Hence $u^{-1} N\left(u s u^{-1}\right) u=N\left(u^{-1}\right)+N(s)+s N\left(u^{-1}\right) s$. Since $W^{\prime \prime}=s W^{\prime \prime} s$, we get

$$
\begin{aligned}
u^{-1} N\left(u s u^{-1}\right) u \cap W^{\prime \prime} & =\left(N\left(u^{-1}\right) \cap W^{\prime \prime}\right)+\left(N(s) \cap W^{\prime \prime}\right)+s\left(N\left(u^{-1}\right) \cap W^{\prime \prime}\right) s \\
& =\emptyset+\{s\}+\emptyset=\{s\}
\end{aligned}
$$

as required.

2.3. Proof of Proposition 1. Suppose $W^{\prime}$ is generated by $x_{1}, \ldots, x_{m}$. Let $W^{\prime \prime}$ be a reflection subgroup of $W$, and $S^{\prime \prime}=\chi\left(W^{\prime \prime}\right)$. We have $x_{i} \in W^{\prime \prime}$ iff $x_{i}$ has some reduced expression $x_{i}=r_{n} \cdots r_{1}$ in $\left(W^{\prime \prime}, S^{\prime \prime}\right)$ iff there is a directed path $e=w_{0}, w_{1}, \ldots, w_{n}=x_{i}$ in $\Omega_{\left(W^{\prime \prime}, S^{\prime \prime}\right)}$ with $w_{j} w_{j-1}^{-1}=r_{j} \in S^{\prime \prime}$ for $j=1, \ldots, n$ iff there is a directed path $e=w_{0}, w_{1}, \ldots, w_{n}=x_{i}$ in $\Omega_{(W, S)}$ with $w_{j} w_{j-1}^{-1}=r_{j} \in S^{\prime \prime}$ for $j=1, \ldots, n$. Note that there are only finitely many directed paths from $e$ to $x_{i}$ in $\Omega_{(W, S)}$, since all vertices $y$ of such a path are in the (finite) Bruhat interval $\left[e, x_{i}\right]$ and the standard length function of $(W, S)$ strictly increases along the path.

Consider the subsets $R$ of $T$ such that $R=\chi(\langle R\rangle)$ and for each $i=1, \ldots, m$, there is some directed path $e=w_{0}, w_{1}, \ldots, w_{n}=x_{i}$ (with $n$ and the $w_{j}$ depending on $i$ ) in $\Omega_{(W, S)}$ with $w_{j} w_{j-1}^{-1} \in R$ for $j=1, \ldots, n$. From above, such sets $R$ are precisely the sets of canonical Coxeter generators of the reflection subgroups of $(W, S)$ which contain $W^{\prime}$. The above remarks also imply that the set of such subsets $R$, when ordered by inclusion, has only finitely many minimal elements, say $R_{1}, \ldots, R_{n}$, and that the reflection subgroups $W_{1}, \ldots, W_{n}$ defined by $W_{i}=\left\langle R_{i}\right\rangle$ for $i=1, \ldots, n$ have the required properties. In fact, any minimal set $R$ as above is a subset of the finite set $T \cap\left\{x y^{-1} \mid x \leq y \leq x_{i}\right.$ for some $\left.i=1, \ldots, n\right\}$, so the $R_{i}$ are effectively computable.

2.4. Recall that a parabolic subgroup of a parabolic subgroup of $W$ is itself a parabolic subgroup of $W$. Further, the intersection of two parabolic subgroups of $W$ is a parabolic subgroup of both of them, and hence also a parabolic subgroup of $W$, by a well-known result of Kilmoyer (see [4, Theorem 2.7.4]; the proof there for the case of finite $W$ readily extends to arbitrary $W$ ). It follows that the intersection $W^{\prime \prime}$ of all parabolic subgroups containing a given finitely generated subgroup $W^{\prime}$ of $W$ is the unique parabolic subgroup $W^{\prime \prime}$ of $W$ of minimal (finite) rank containing $W^{\prime}$. One calls $W^{\prime \prime}$ the parabolic closure of $W^{\prime}$. Note that $W^{\prime \prime}$ is a parabolic subgroup of any (finite rank) standard parabolic subgroup of $W$ which contains $W^{\prime}$.

2.5. Proof of Proposition 2. Suppose first that $W^{\prime \prime}$ is a parabolic subgroup of $W$. The remarks of the preceding subsection show that $W^{\prime \prime}$ is a parabolic subgroup of $W_{J}$, so $w W^{\prime \prime} w^{-1}=W_{K}$ for some $K \subseteq J$ and $w \in W_{J}$. Write $w=y z$ where $z \in W^{\prime \prime}$ and $N\left(y^{-1}\right) \cap W^{\prime \prime}=\emptyset$. Then $W_{K}=y W^{\prime} y^{-1}$ so by Lemma $1, K=$ $\chi\left(W_{K}\right)=y \chi\left(W^{\prime}\right) y^{-1}=\left\{y c_{1} y^{-1}, \ldots, y c_{n} y^{-1}\right\}$. Letting $y c_{i} y^{-1}=s_{i} \in K$, we have $y\left(c_{1} \cdots c_{n}\right) y^{-1}=s_{1} \cdots s_{n}$ as required.

Conversely, suppose $s_{1}, \ldots, s_{n}$ in $J$ are pairwise distinct and $w\left(c_{1} \cdots c_{n}\right) w^{-1}=$ $s_{1} \cdots s_{n}=c$. We may assume without loss of generality that $W$ is realized in its 
MATTHEW DYER

standard reflection representation (see e.g. $[9, \mathrm{Ch} 5]$ ) on a real vector space $V$ with standard symmetric bilinear form, with associated root system $\Phi$ and linearly independent simple roots $\Pi$. For $\alpha \in \Phi$, we let $s_{\alpha} \in W$ denote the orthogonal reflection in $\alpha$. By enlarging $V$ and extending the form if necessary, we may assume that the form (?|?) on $V$ is non-degenerate.

Let $s_{i}=s_{\alpha_{i}}$ and $c_{i}=s_{\beta_{i}}$ where $\alpha_{i} \in \Pi$ and $\beta_{i} \in \Phi$. Using non-degeneracy of the form, it is easy to see that $\operatorname{Im}(e-c)=V^{\prime}:=\sum_{i=1}^{n} \mathbb{R} \alpha_{i}$ is $n$-dimensional. On the other hand, if $\gamma_{i}, \ldots, \gamma_{m} \in \Phi$ with $c=s_{\gamma_{1}} \cdots s_{\gamma_{m}}$, then $\operatorname{Im}\left(e-s_{\gamma_{1}} \cdots s_{\gamma_{m}}\right) \subseteq$ $\sum_{i=1}^{n} \mathbb{R} \gamma_{i}$ is at most $m$-dimensional. Hence if $c=s_{\gamma_{1}} \cdots s_{\gamma_{m}}$ with $m \leq n$ we get that $m=n$ and $\gamma_{1}, \ldots, \gamma_{n}$ is a $\mathbb{R}$-basis of $V^{\prime}$. It is well known (see e.g. [3, Proposition 3.3]) that $\gamma_{i} \in V^{\prime}$ implies $s_{\gamma_{i}} \in W_{K}$ for $i=1, \ldots, n$, where $K=\left\{s_{1}, \ldots, s_{n}\right\}$.

Applying the preceding paragraph with $\gamma_{i}=w\left(\beta_{i}\right)$ shows that $\gamma_{i} \in V^{\prime}$ and $s_{\gamma_{i}}=$ $w c_{i} w^{-1} \in W_{K}$ for $i=1, \ldots, n$. Write $w=y z$ where $z \in W^{\prime \prime}$ and $N\left(y^{-1}\right) \cap W^{\prime \prime}=\emptyset$. Since $W^{\prime \prime}=\left\langle z c_{i} z^{-1} \mid i=1, \ldots, n\right\rangle$, it follows that $y W^{\prime \prime} y^{-1} \subseteq W_{K}$. Choose a reduced expression $p=r_{1} \cdots r_{k}$ for $p:=z c_{1} \cdots c_{n} z^{-1}$ in $\left(W^{\prime \prime}, \chi\left(W^{\prime \prime}\right)\right)$. Then there is a directed path $e, r_{k}, r_{k-1} r_{k}, \ldots, r_{1} \cdots r_{k-1} r_{k}=p$ in $\Omega_{\left(W^{\prime \prime}, \chi\left(W^{\prime \prime}\right)\right)}$. By Lemma 1, conjugating by $y$ gives a directed path

$$
e, y r_{k} y^{-1}, y r_{k-1} r_{k} y^{-1}, \ldots, y r_{1} \cdots r_{k-1} r_{k} y^{-1}=y p y^{-1}=s_{1} \cdots s_{n}
$$

in $\Omega_{\left(y W^{\prime \prime} y^{-1}, \chi\left(y W^{\prime \prime} y^{-1}\right)\right)}$. The last path is also a directed path in $\Omega_{(W, S)}$. Now since the $s_{i}$ are pairwise distinct, any path $e=q_{0}, q_{1}, \ldots, q_{l}=s_{1} \cdots s_{n}$ in $\Omega_{(W, S)}$ has $l=n$ and satisfies $\left\langle q_{i} q_{i-1}^{-1} \mid i=1, \ldots, n\right\rangle=\left\langle s_{1}, \ldots, s_{n}\right\rangle$ (for example, by induction on $n$ ). Hence $k=n$ and $\left\langle y r_{i} y^{-1} \mid i=1, \ldots, n\right\rangle=W_{K}$. Thus, $y W^{\prime \prime} y^{-1} \supseteq W_{K}$. Since the reverse inclusion has previously been established and $z \in W^{\prime \prime}$, we get $w W^{\prime \prime} w^{-1}=y W^{\prime \prime} y^{-1}=W_{K}$, completing the proof.

2.6. Proof of Corollary 3. Corollary 3 follows immediately from the remarks in 2.4 since they imply that the parabolic closure of $W^{\prime}$ must be one of the subgroups $W_{i}$ in Proposition 1.

\section{REFERENCES}

[1] Patrick Bahls. The isomorphism problem in Coxeter groups. Imperial College Press, 2005.

[2] N. Bourbaki. Éléments de mathématique. Fasc. XXXIV. Groupes et algèbres de Lie. Chapitre IV: Groupes de Coxeter et systèmes de Tits. Chapitre V: Groupes engendrés par des réflexions. Chapitre VI: systèmes de racines. Actualités Scientifiques et Industrielles, No. 1337. Hermann, Paris, 1968.

[3] Brigitte Brink and Robert B. Howlett. Normalizers of parabolic subgroups in Coxeter groups. Invent. Math., 136(2):323-351, 1999.

[4] Roger W. Carter. Finite groups of Lie type. Wiley Classics Library. John Wiley \& Sons Ltd., Chichester, 1993.

[5] Arjeh Marcel Cohen. Recent results on Coxeter groups. In Polytopes: abstract, convex and computational (Scarborough, ON, 1993), volume 440 of NATO Adv. Sci. Inst. Ser. C Math. Phys. Sci., pages 1-19. Kluwer Acad. Publ., Dordrecht, 1994.

[6] Matthew Dyer. Hecke Algebras and Reflections in Coxeter Groups. PhD thesis, Univ. of Sydney, 1987.

[7] Matthew Dyer. Reflection subgroups of Coxeter systems. J. Algebra, 135(1):57-73, 1990.

[8] Matthew Dyer. On the "Bruhat graph" of a Coxeter system. Compositio Math., 78(2):185$191,1991$.

[9] James E. Humphreys. Reflection groups and Coxeter groups, volume 29 of Cambridge Studies in Advanced Mathematics. Cambridge University Press, Cambridge, 1990.

[10] Daan Krammer. The conjugacy problem for Coxeter groups. PhD thesis, Universiteit Utrecht, 1994. 
Department of Mathematics, 255 Hurley Building, University of Notre Dame, Notre DAME, INDiAna 46556, U.S.A.

E-mail address: dyer.1@nd.edu 